\title{
Genetic markers of prostate cancer aggressiveness
}

C urrent prognostic tools lack sufficient accuracy to identify the subset of patients with aggressive prostate cancer who are at high risk of recurrence and death. Besides conventional clinical parameters, some genetic alterations including rearrangements in ETS transcription factor genes-the most common of which is the TMPRSS2-ERG fusion-have been linked to poor prognosis in this disease. Now, three new papers have shed light on potential genetic markers for aggressive prostate cancer.

Combining data on ETS gene rearrangements and loss of the tumor suppressor gene PTEN predicts risk of death from prostate cancer. So says a team of researchers working on behalf of the Transatlantic Prostate Group. "Using gene status, we identified a group of patients that did very well, and a group of patients that did very badly," says lead author Alison Reid from the UK Institute of Cancer Research. "These results could have important implications for deciding which patients should be conservatively or aggressively treated."

The investigators used fluorescent in situ hybridization to assess ETS gene rearrangements (in ERG and ETV1) and $P T E N$ loss in prostate tissue specimens from 308 men with incidentally detected prostate cancer who were managed conservatively.

The majority of patients (54\%) had no ETS gene rearrangement and no evidence of PTEN gene loss. These patients demonstrated a favorable disease-specific survival rate $(85.5 \%$ at 11 years). By contrast, patients without ETS rearrangement, but with PTEN gene loss ( $6 \%$ of the population) demonstrated very poor disease-specific survival (13.7\% at 11 years). Patients who had evidence of ETS rearrangements had intermediate 11-year disease-specific survival rates $(59.8 \%$ for ETS rearrangements without PTEN loss, and $41.0 \%$ for ETS rearrangements with PTEN loss).

In addition to predicting clinical outcomes, the researchers highlight that their results could have important implications for the stratification of patients in clinical trials. "An imbalance in the proportions of patients with different PTEN and ETS gene status between study arms could falsely influence trial outcome," observes Reid. "ETS gene rearrangement and PTEN gene loss status should now be prospectively incorporated into predictive models, to establish whether

predictive performance is improved."

In a separate study, researchers from the USA and Canada have characterized molecular alterations associated with TMPRSS2-ERG-positive cancer, and identified genetic markers of biochemical recurrence in radical prostatectomy specimens from patients with prostate cancer. If validated, such markers might have clinical utility in prognosis and therapeutic decision-making.

Using a panel of 502 cancer-related genes in tissue samples from 139 men and a validation cohort of 596 men, the investigators identified five genes associated with recurrence and four with nonrecurrence. A nine-gene expression score was a significant predictor of biochemical recurrence on multivariate analysis, which included Gleason score and TMPRSS2-ERG fusion status.

They also identified 15 genes that were differentially regulated in TMPRSS2-ERGpositive versus TMPRSS2-ERG-negative disease, and these were validated in an independent patient cohort. The ETS gene $E R G$ showed the most significant degree of differential expression. The authors conclude that TMPRSS2-ERGpositive tumors have a distinct molecular metabolism, which is replicated in multiple cohorts. As these fusions are potentially associated with increased rates of recurrence, these additional 15 genes might be implicated in prostate cancer progression.

Finally, Jianfeng Xu and colleagues from institutions in the USA and Sweden have identified a potential single nucleotide polymorphism (SNP) marker of aggressive

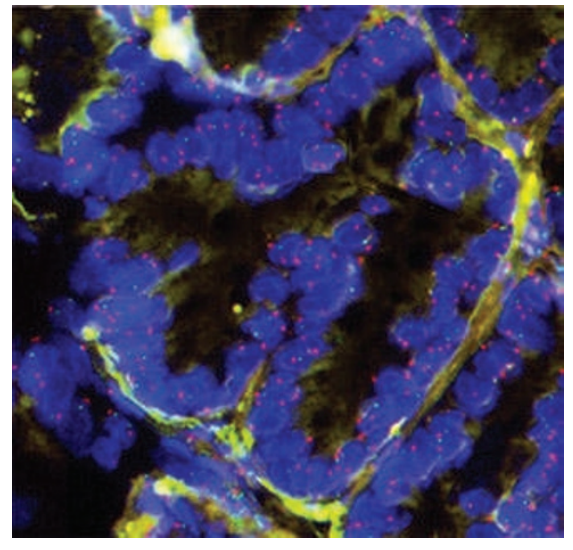

Fluorescent in situ hybridization showing heterozygous loss of PTEN. Modified, with permission from Reid, A. H. M. et al. Br. J. Cancer doi:10.1038/sj.bjc.6605554 @ 2010, Nature Publishing Group.

prostate cancer. They compared SNP frequencies in a total of 17,034 patients defined as having either more-aggressive or less-aggressive disease. The TT genotype of the SNP rs4054823 was significantly more common in patients with more-aggressive disease; this SNP met the criteria for genome-wide significance. The authors conclude that gene variants predisposing to progression and recurrence exist in the human genome, and that such variants have potential clinical utility as markers of aggressive disease.

Studies such as these now indicate that prostate cancer can be characterized at the molecular level to yield clinically informative data. This information holds great potential for distinguishing patients who need aggressive treatment from those who can be managed conservatively.

\section{Nick Groves-Kirkby}

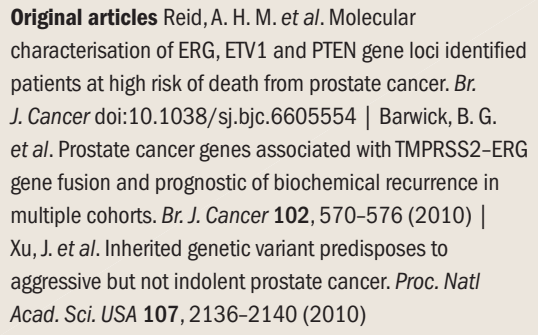

